\title{
NOTES AND GOMMENT
}

\section{Infra-red Observation and Cinematography of Microcrustacea}

The purpose of this note is to present a simple illumination system for observation or photography of small semi-transparent animals such as microcrustacea. This system of illumination makes semi-transparent organisms appear as dark silhouettes on a light field. Use of the near infra-red spectrum permits observation and photography of experimental animals in extremely dim light or in total darkness. An observation and recording technique which is independent of ambient visible intensity is a distinct advantage in the study of dark adaptation or general behavior patterns of many pelagic plankters which behave very differently in different light intensities. Infra-red movies are used to establish the existence of various behavior patterns by the usual correlations of linear and angular velocities with intensities and gradients of the several physical and chemical parameters of the environment.

The illumination system is shown in Figure 1. It is directly comparable to Köhler illumination with the microscope. The lens $B$ performs the same function as the microscope condenser, with the result that the light source is seen to be the same size as the condensing lens.

The light source $A$ is a Westinghouse 500 -watt photospot RSP-2 immersed in a mineral oil bath for absorption of the unwanted longer infra-red wavelengths largely responsible for heating. Visible radiation is excluded by use of a six-inch square black glass filter $5 / 16$ of an inch thick. ${ }^{1}$ Black Carrara glass filters compare favorably in transmission with commercially available glass infra-red filters and appear considerably more resistant to fracture from heat or mechanical shock. The black glass filter is clamped to the oil bath against an " $O$ " ring seal or any other suitable gasket for retaining the oil in the bath. An oil expansion reservoir must be provided since the oil becomes quite hot during the operation of the lamp. The oil reservoir should be three inches in diameter and about six inches high. The hole into the oil bath from the expansion reservoir should be about three inches in diameter as a safety precaution in the event of implosion of the lamp. For long periods of operation such as with an infra-red viewing device, a copper cooling coil connected to the coldwater tap should be included in the oil bath. Satisfactory intensities in the photographic infra-red spectral band $(7500-12,500 \AA)$ are obtained from this light source when the lamp is operated at 3250 degrees Kelvin.

The light source $A$ in Figure 1 is located at one focal point of the condensing lens. The aquarium of experimental animals $C$ is placed next to the

1 Black Carrara glass used for building facings and manufactured by Pittsburgh Glass Co., Pittsburgh, Pennsylvania. condensing lens, and the camera or observers eye is placed at the other focal point $D$. The effect of such a system of illumination is to image the light source at the camera lens or observers eye where the imaging lens is scen brightly illuminated with the aquarium and zooplankton silhouetted against the brightly lighted field of the lens. A 14 by 18 inch plastic Fresnel lens having a focal length of two feet is used for the condensing lens. ${ }^{2}$

If the aquarium is placed on the same side of the condensing lens as the cumera or observer the experimental animals appear natural size but if the aquarium is placed on the opposite side the images of the experimental animals will be magnified in accordance with the well known ratio

$$
\frac{\text { size of image }}{\text { size of object }}=\frac{\text { distance from lens to image }}{\text { distance from lens to object }}
$$

Since the aquarium is quite close to the lens the images will appear virtual and erect.

A condensing lens is more efficient than frosted glass for background illumination since it gathers light from a large solid angle and focuses it on the camera lens, while frosted glass gives only the limited forward scatter of light incident upon it. In our experiments the light intensity at the camera lens is 10,000 times as intense with a condensing lens as with frosted glass. The distribubution of intensity in the forward scatter from ground glass is responsible for the non-uniform exposures of vignetting obtained when frosted glass is used as a diffusing background. Opal glass is only a little better. Neither opal glass nor frosted glass is practical as a background for wide areas close up to the camera.

Fxposure of Eastman Kodak High Speed Infra Red Movic film at 16 frames per second with a stop opening of $f \mathbf{2 . 5}$ gives an optical density of 0.9 with normal development in Eastman Kodak Microdol. Such organisms as the almost completely transparent cladoceran, Leptodora kindtii, or the relatively transparent mysid shrimp, Mysis relicta, which are usually seen best by their own scattered light or through crossed polaroids can be photographed as silhouettes.

It is important to know that the infra-red radiation employed in this method does not stimulate the experimental animals. Although it is impossible to state categorically that infrared fails to stimulate it is possible to state operationally that infra-red produces no unique behavior patterns, nor does it alter any existing behavior pattern thus far tested. Presumably any alteration of an existing behavior pattern must be achieved by some alteration in linear or angular velocity. We have, therefore checked for such changes.

2 Edmund Scientific Co., Barrington, N. J. 


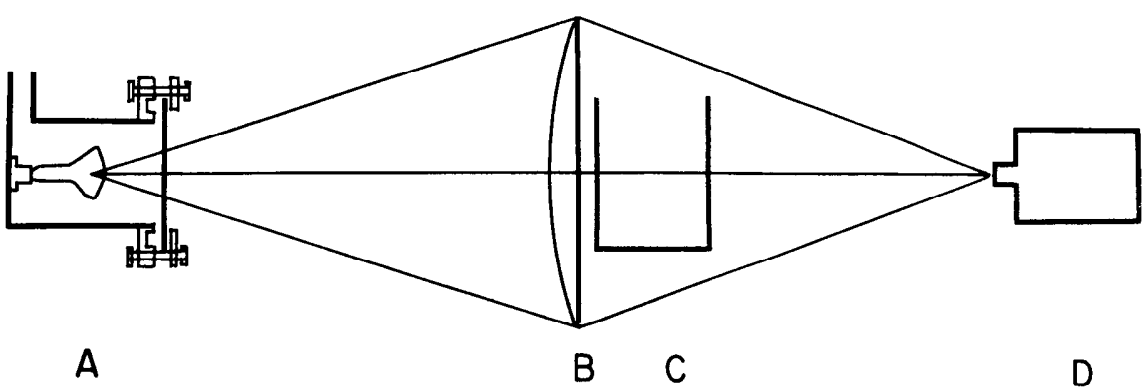

Fig. 1. Diagram of the illumination system: $A$ is the light source; $B$ is a $14 \mathrm{x} 18$ inch plastic Fresnel lens; $C$ is the aquarium containing experimental animals; $D$ is a movie camera.

To test for changes in linear velocity we invoked the null hypothesis that there is no significant difference in linear velocities in white light with and without infra red. Such a null hypothesis is amenable to the statistics of small samples.

The experiment was performed as follows. A population of Daphnia magna adapted to a given intensity of white light was continuously photographed with a movie camera for a period of thirty seconds. The animals were illuminated with white light for the first 15 seconds and with white light plus infra-red for the last 15 seconds. Analysis of the photographic record thus obtained shows an average linear velocity of $0.201 \mathrm{~cm} / \mathrm{sec}$. in white light and $0.196 \mathrm{~cm} / \mathrm{sec}$. in white plus infra-red. By the use of the Wilcoxon Sum for ranked differences of paired data, it was concluded that the mean differences of velocities are not significantly different from one another within the limits of the data.

Analysis of the same photographic record for angular velocities reveals an average angular velocity of 5 degrees/sec. in white light and 6 degrees/sec. in white plus infra-red. Again the use of the Wilcoxon Sum for the paired angular velocities indicates that they are not significantly different from each other within the limits of the data. The failure of Daphnia magna to respond to infra-red should not be extrapolated to other organisms without appropriate testing.

Finally, many behavior patterns are statistical in nature and are not readily observable over short periods of time. Such statistical behavior typically results in a net movement of a population in one direction to produce an aggregation at some given locus. The infra-red radiation employed in these studies whether with or without visible light failed to produce any aggregations of the population either toward or away from the source, or at the bottom of the container or at the surface of the water in a period of 2 hours.

Fidward R. Baylor ${ }^{3}$

Zoology Department, University of Michigan, Ann Arbor, Michigan.

\section{The Use of Carbon Dioxide to Construct $\mathrm{pH}$ Curves for the Measurement of Productivity ${ }^{1}$}

\section{INTRODUCTION}

In recent years there has been increasing use of $\mathrm{pH}$ measurement for the determination of carbon metabolism in natural waters and in closed containers with living organisms (Verduin 1951, 1956a, 1956b; Jackson and McFadden 1954; Odum 1957a, 1957b; McQuate 1956; Park et al. 1958). A graph of $\mathrm{pH}$ against $\mathrm{CO}_{2}$ is constructed for the particular water. The data for this graph are obtained either experimentally or by theoretical computation from alkalinity. Then the observed $\mathrm{pH}$ changes are converted into $\mathrm{CO}_{2}$ changes by means of the graph. For some time, it has been apparent to us that one of the customary experimental procedures in this method leads to con-

1 These studies were aided by the National Science Foundation through grant NSF G3978 on Ecological Microcosms. siderable error. The purpose of this note is to point out the error and suggest a better procedure applicable to both sea water and fresh water based on the use of $\mathrm{CO}_{2}$ water.

In fresh water the theoretical equilibrium equations of Moore (1939) were used by Dye (1944) to compute nomograms so that with a known alkalinity, $\mathrm{pH}$ changes can be converted to $\mathrm{CO}_{2}$ changes without actually determining the $\mathrm{pH}$ $\mathrm{CO}_{2}$ curve experimentally. In this work the alkalinity is determined by titration with a strong acid to approximately $\mathrm{pH}$ 4.5. Either a color indicator (such as methyl purple or methyl orange) or a $\mathrm{pH}$ electrode is used to locate the end point.

In sea water the constants in the equilibrium

${ }^{3}$ Present address: Woods Hole Oceanographic Institution, Woods Hole, Massachusets. 